\title{
Design and Efficient Soluble Expression of a Sweet Protein, Brazzein and Minor-Form Mutant
}

\author{
Jin-Ju Lee, Ji-Na Kong, Hyun-Dong Do, Dong-Hyeon Jo, and Kwang-Hoon Kong* \\ Laboratory of Biomolecular Chemistry, Department of Chemistry, College of Natural Sciences, Chung-Ang University, \\ Seoul 156-756, Korea. *E-mail: khkong@cau.ac.kr \\ Received September 9, 2010, Accepted September 27, 2010
}

Key Words: Brazzein, Periplasmic secretion, Site-directed mutagenesis, Soluble expression, Sweet protein

The demand for non-calorigenic protein-based sweeteners with favorable taste properties is high. Most proteins are tasteless and flavorless, while some proteins elicit a sweet-taste response on the human palate. ${ }^{1}$ Seven sweet-taste proteins derived from a variety of plants (or rarely from animals) were identified as eliciting a sweet-taste response: thaumatin; monellin; mabinlin; brazzein; neoculin; miraculin; egg white lysozyme. Among them, brazzein possessed better $\mathrm{pH}$ and thermal stabilities and a pleasant sweet taste profile. Brazzein was isolated from the fruit of the West African Pentadiplandra brazzeana Baillon plant. $^{2}$ It is a single-chain polypeptide consisting of 54 amino acid residues, with a corresponding molecular mass of approximately $6.5 \mathrm{kDa}$. Brazzein is heat-stable, with its sweetness remaining after heating at $98{ }^{\circ} \mathrm{C}$ for $2 \mathrm{~h}$ and $80{ }^{\circ} \mathrm{C}$ for $4.5 \mathrm{~h}$ at a pH range of $2.5-8$. This stability may be due to four intramolecular disulfide bonds and the absence of no-free sulfhydryl groups within the molecule. Brazzein water solubility is at least $50 \mathrm{mg} / \mathrm{mL}$. From the point of view of its sweetness, heat stability, high water solubility, and minimum molecular weight, brazzein is currently the superior protein sweetener.

Extraction of brazzein from its natural source is expensive and, therefore, not applicable. To date, brazzein has been produced in E. coli, ${ }^{3,4}$ maize, ${ }^{5}$ and Lactococcus lactis. ${ }^{6}$ These heterologous productions of brazzein are often complicated by the fact that the protein contains four disulfide bridges and requires a specific $N$-terminal sequence. Previous studies for the expression of brazzein from Escherichia coli report that the recombinant brazzein expressed in the cytoplasm of Escherichia coli exists in an insoluble form, involving several steps with a low overall yield: expression as a fusion protein, denaturation and renaturation, oxidation of the cysteines, and cleavage by cyanogen bromide or SUMO protease at an engineered methionine adjacent to the desired $N$-terminus. To improve production levels of the recombinant soluble brazzein, in the present study, we established a new strategy using the pelB leader sequence of $E$. coli for brazzein expression. Secretory production of the recombinant proteins by $E$. coli has several advantages over intracellular production as inclusion bodies. In most cases, targeting protein production to the periplasmic space facilitates downstream processing, folding, and in vivo stability, enabling production of soluble and biologically active proteins at a reduced process cost. When the protein had multiple intramolecular disulfide bonds, it was difficult to achieve proper folding in the cytosol of E. coli since it is highly reducing environment.
Nevertheless, the secretion of this protein into the periplasmic space in $E$. coli gives a better chance of proper folding due to the increased oxidizing conditions in this extracellular compartment. Besides the formation of correct disulfide bonds, production into the periplasmic space can also ease purification. ${ }^{7,8}$ Thus, we designed a gene with a pelB signal sequence adjacent to the $N$-terminal for soluble protein production in the periplasmic space and synthesized the gene that incorporated codons optimal for protein production in E. coli (Fig. 1). The synthetic brazzein gene was cloned into $\mathrm{pET}-26 \mathrm{~b}$ to improve production levels of the recombinant soluble brazzein by periplasmic secretion. The resulted expression vector, $\mathrm{pET}$-Brazzein was transformed the E. coli strain BL21 Star (DE3).

Brazzein purified from its natural source consists of a major form $(\sim 80 \%)$ and minor form $(\sim 20 \%){ }^{2}$. The major form contains a pyroglutamate at its $N$-terminus, while the minor form lacks this $N$-terminal pyroglutamate. The major form is 500 to 2,000 times sweeter than sucrose. Conversely, the minor form has nearly twice the sweetness of the major form. To make the minor-form mutant that lacked a $N$-terminal methionine, a starting codon in the Nco I site of the pET-Brazzein was deleted by site-

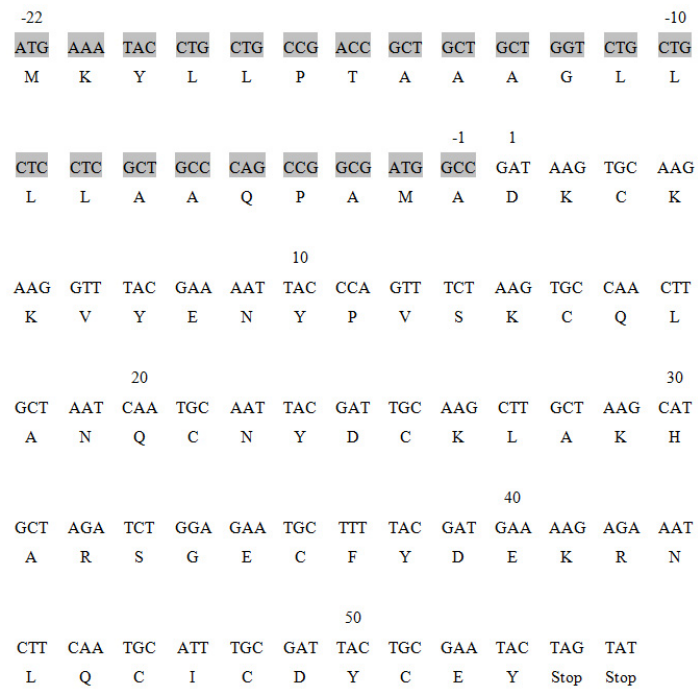

Figure 1. The DNA sequence of the synthesized brazzein gene encoding 53 amino acids that combined with the pelB leader sequence and amino acid sequence of the resulted recombinant brazzein. The sequence ends with two TAG and TAA termination codons. Gray box ( ) indicates the sequence of the pelB leader sequence. 
Table 1. Comparison of the sweet taste properties of sucrose, the wild-type brazzein (WT-Brazzein) and the minor-form mutant (MFMutant)

\begin{tabular}{cccccc}
\hline \multirow{2}{*}{$\begin{array}{c}\text { Sweet tasting } \\
\text { molecule }\end{array}$} & $\begin{array}{c}\text { Experimental taste } \\
\text { threshold }\end{array}$ & & \multicolumn{2}{c}{$\begin{array}{c}\text { Sweetness in comparison } \\
\text { to sucrose }\end{array}$} \\
\cline { 2 - 3 } \cline { 5 - 6 } & $\left(\mathrm{g}(100 \mathrm{~mL})^{-1}\right)$ & $(\mu \mathrm{M})$ & & $(\mathrm{g} / \mathrm{g})$ & $($ molecule $)$ \\
\hline Sucrose & 1.0 & 58,000 & & 1 & 1 \\
WT-Brazzein & 0.0025 & 3.94 & & 800 & 14,848 \\
MF-Mutant & 0.0011 & 1.71 & & 1840 & 34,150 \\
\hline
\end{tabular}

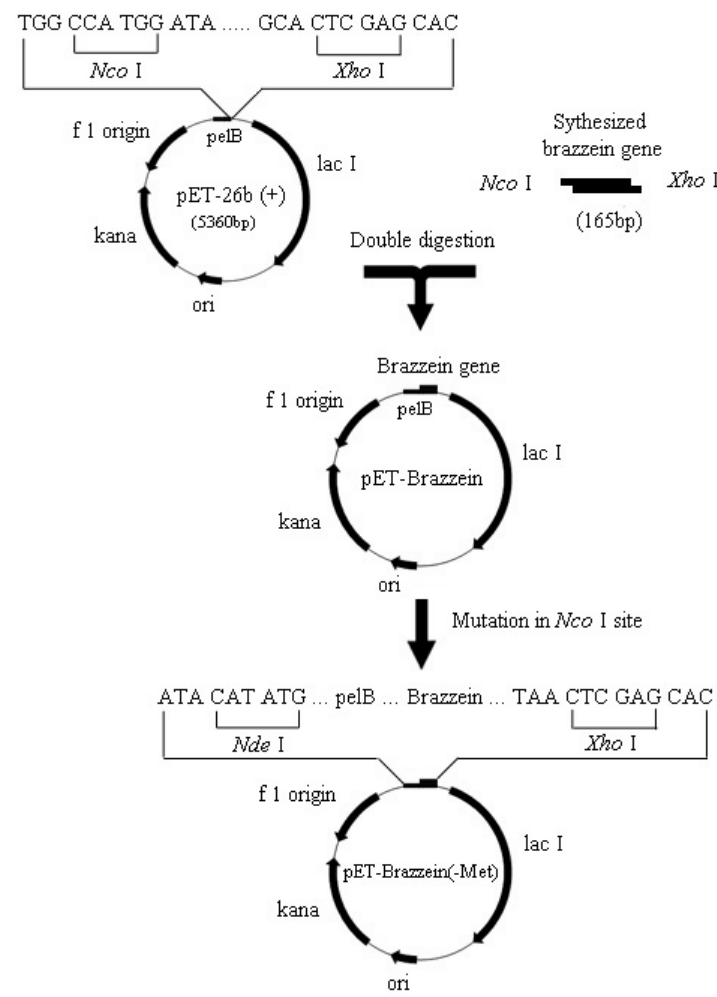

Figure 2. Construction of the expression vectors, pET-Brazzein and pET-Brazzein(-Met). The synthetic brazzein gene was inserted into the pET-26b vector with both Nco I and Xho I restriction sites and deleted a starting codon in the Nco I site by site-directed mutagenesis to make the minor-form brazzein, which lacked an $N$-terminal methionine.

directed mutagenesis (Fig. 2). The resulted expression vector, pET-Brazzein(-Met), was transformed the E. coli strain BL21 Star (DE3).

In an attempt to improve expression levels of the soluble form, different expression conditions were tested (optical density $\mathrm{A}_{600}$ at induction, IPTG concentration, temperature of growth, E. coli strain). For maximum yield of the soluble protein, E. coli BL21 Star (DE3) cells harboring pET-Brazzein and pET-Brazzein (-Met) were grown at $30^{\circ} \mathrm{C}$ for $10 \mathrm{~h}$. In addition, the recombinant wild-type brazzein and minor-form mutant were strongly expressed without adding an inducer IPTG and seemed to be expressed by basal expression under our expression system. The recombinant wild-type brazzein and minor-form mutant were efficiently produced in the soluble form into the periplasm at an amount approximately $80-90 \%$ of the total periplasmic pro-

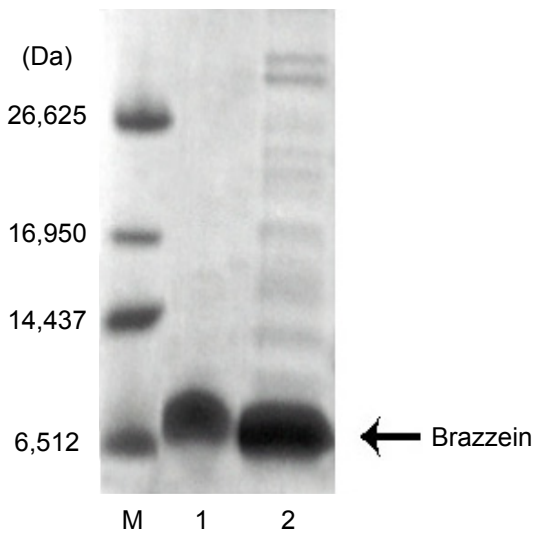

Figure 3. SDS-PAGE analysis at different stages of purification. Lane $\mathrm{M}$, molecular weight standard marker; lane 1, purified recombinant wild-type brazzein after periplasmic fractionation and heat treatment; lane 2, periplasmic fraction in BL21 Star (DE3) with pET-Brazzein.

teins. They were purified to yield approximately $6.7 \mathrm{mg} / \mathrm{L}$, using the periplasmic fractionation and heat treatment. The purities of the recombinant wild-type brazzein and minor-form mutant were confirmed by SDS-PAGE and HPLC. The purified brazzein appeared as a single band on SDS-PAGE with an apparent $\mathrm{M}_{\mathrm{r}}$ of 6,500 Da (Fig. 3); the elution times for the folded recombinant brazzeins were $11 \pm 0.5 \mathrm{~min}$, as denoted by RP-HPLC (data not shown).

To identify the successive secretory production of the designed recombinant brazzeins into the periplasmic space of $E$. coli, the $N$-terminal amino acids of the purified recombinant brazzeins were determined by automated Edman degradation. The $N$-terminal amino acid sequences of the purified recombinant wildtype brazzein and minor-form mutant were M-D-K-C-K-K-VY-E-N and D-K-C-K-K-V-Y-E-N corresponded to that deduced from the minor form of the brazzein gene, respectively. These results revealed that the brazzeins expressed using the pelB leader sequence were localized in the periplasmic fraction of the cell and the pelB leader sequence cut with signal peptidase during protein exit out from cytosol to periplasm. ${ }^{9}$

Compared to the sucrose solutions of different concentrations, the intensities of sweetness of the purified recombinant brazzeins were as potent as the natural one. The intensity of sweetness of the recombinant wild-type brazzein was approximately 800 times sweeter than sucrose on a weight basis, approximately 14,848 times sweeter on a molar basis. On the other hand, the intensity of sweetness of the recombinant minor-form mutant was approximately 1,840 times sweeter than sucrose on a weight basis, approximately 34,150 times sweeter on a molar basis, and equivalent to that of the minor form isolated from fruit. This indicates that the $\mathrm{N}$-terminal deletion mutation increases sweetness, and the $N$-terminal region is involved in the sweetness of brazzein. Assadi-Porter et al. ${ }^{3,10}$ also reported that Asp2Asn replacement resulted in decreased sweetness, whereas increasing the size of the side chain (Asp2Glu) while maintaining the charge leads to little or no change in sweetness. Taken together, we suggest that Asp2 residue in $N$-terminus of brazzein plays a role in sweetness and the charge at this position is essential to function. 
The bacterial system is ideal for its ease of rapid genetic manipulation, such as site-directed mutagenesis. Conventional $\mathrm{pET}$ vectors are generally successful for the expression of soluble foreign proteins. However, the yields with this expression system of small proteins containing multiple disulfide bridges, such as brazzein, are frequently low. Previous studies to express genetically optimized synthetic brazzein in $E$. coli involved expression as an inclusion body or as fusion proteins with staphylococcal nuclease and His-SUMO, but required several purification steps with low overall yields. ${ }^{3,4}$ Accordingly, proteins of this kind often are expressed in yeast or insect cells, which provide a eukaryotic environment. Previous attempts to express genetically optimized synthetic brazzein in yeast have proven unsuccessful. ${ }^{11,12}$ An alternative approach is to produce the target protein as a secretory protein. In this study, we have shown the secretory system to be an excellent method of producing the soluble form of brazzein in $E$. coli at high yield. Moreover, the pelB signal peptide used in this study was effective for directing recombinant brazzein to be secreted into the peripheral space, but not into the culture. In addition, the recombinant brazzein could be purified by incubating the periplasmic fractions at high temperatures. The new expression method in this study has three major advantages over the $E$. coli expression systems developed earlier: (1) the pET-Brazzein and pET-Brazzein(-Met) expression systems based on the periplasmic secretion do not require solubilization and refolding; (2) the purification procedure is simple and quick; (3) the recombinant proteins do not require protease cleavage or $\mathrm{CNBr}$ cleavage. Thus, the new protocol provides a higher yield of brazzein with less time and effort.

In summary, we designed the synthetic gene encoding brazzein, a sweet protein based not only on the biased codons of Escherichia coli, so as to optimize its expression, but also on the periplasmic secretion for expression in an active, soluble form. To make the minor-form mutant, a starting codon in the gene was deleted by site-directed mutagenesis. The expressed soluble recombinant wild-type brazzein and minor-form mutant were purified by periplasmic fractionation and heat treatment to give a higher yield. The identity and purity of the recombinant proteins were confirmed by $N$-terminal amino acid analysis, taste testing, SDS-PAGE, and HPLC. The purified wild-type brazzein and minor-form mutant had approximately 800 and 1,840 times sweeter than sucrose on a weight basis, respectively. This suggests that the $N$-terminal region of brazzein is involved in the sweetness. The current expression and purification protocol should prove useful for the production of large amounts of active and soluble brazzein to study the relationship between the structure and sweetness of brazzein, as well as the interaction between sweet-tasting proteins and their putative receptors by site-directed mutagenesis and structural analysis.

\section{Experimental Section}

Design and synthesis of the brazzein gene. The GenScript's OptimumGene ${ }^{\mathrm{TM}}$ Gene Design system (Piscataway, NJ, USA) was used in designing the synthetic gene, which was based on the amino acid sequence of naturally occurring brazzein ${ }^{2}$ and optimized with a codon usage and a stable predicted mRNA secondary structure to achieve the highest possible levels of productivity in E. coli expression systems (Fig. 1).

Construction of the brazzein expression vector and site-directed mutagenesis. The synthetic brazzein gene was cloned into an expression vector pET-26b(+) (Novagen, Madison, WI, USA) with a T7 promoter using Nco I and Xho I sites. The brazzein gene was localized just after a pelB leader sequence in the expression vector to secret recombinant brazzein into the periplasm of the E. coli (Fig. 1). In addition, a starting codon (Met) in the Nco I site was also deleted with site-directed mutagenesis, since the amino acid sequence of natural brazzein lacked a $N$-terminal methionine (Fig. 2). The oligonucleotide primers used for the site-directed mutagenesis follow: primer1,5'-GCGCGGGGGCGCGGCCAGCCGAAG-3'; primer-2, 5'-CTTCGGCTGGCCGCGCC CCCGCGC-3'. Mutagenesis was performed according to the protocol of a Mutant ${ }^{\mathrm{TM}}$-Super Express Km kit (Takara Shuzo Co., Otsu, Shiga, Japan). Construction of a single-stranded DNA template for mutagenesis and confirmation of mutation were performed as described in a previous paper. ${ }^{13}$ The resulting vectors were designated $\mathrm{pET}$ Brazzein and pET-Brazzein(-Met) and used to transform the E. coli strain BL21 Star (DE3). The colony containing the appropriate insert was identified by DNA sequencing (COSMO Genetech, Seoul, Korea).

Expression and purification of recombinant brazzeins. The E. coli strain BL21 Star (DE3) harboring each of the constructed plasmids was grown in LB broth containing kanamycin (30.0 $\mu \mathrm{g} / \mathrm{mL})$ at 30 or $37^{\circ} \mathrm{C}$ and induced at $\mathrm{OD}_{600}=0.4$ with $0-1.0$ $\mathrm{mM}$ isopropyl- $\beta$-D-thiogalactopyranoside (IPTG) for $4-12 \mathrm{~h}$. The induced cells were harvested by centrifugation at $8,000 \mathrm{~g}$ for $10 \mathrm{~min}$ at $4{ }^{\circ} \mathrm{C}$. The periplasmic extract was prepared by the osmotic shock method. ${ }^{14,15}$ The supernatant, hereafter termed the periplasmic fraction, was subjected to thermal treatment at $80{ }^{\circ} \mathrm{C}$ for $1 \mathrm{~h}$ and centrifuged at $40,000 \mathrm{~g}$ for $60 \mathrm{~min}$ at $4{ }^{\circ} \mathrm{C}$. The supernatant was dialyzed against the distilled water and freeze-dried. The final freeze-dried solid brazzeins were white in appearance. The purified proteins were used for the next experiment. Protein concentration was determined by the BCA assay reagent (Pierce Chemical Co., Rockford, IL, USA) using bovine serum albumin as the standard protein.

Measurement of sweet-tasting activity. The sweet-tasting activity of the recombinant brazzeins were assayed by sensory analysis using double blind taste test of twenty people. The purified recombinant brazzeins were lyophilized and dissolved in water $(1.0 \mathrm{mg} / \mathrm{mL})$. The brazzein solutions were prepared at the following concentration: $0.5,1.0,2.0,3.0,4.0,5.0,6.0$, $7.0,8.0,9.0$, and $10.0 \mu \mathrm{g} / \mathrm{mL}$. A $1 \%$ sucrose solution was prepared for comparison as $1 \%$ sucrose is the lowest concentration detectable by humans. The taste panel consisted of ten females and ten males of reported good health and normal sense of taste. Two-hundred-microliter samples were applied to the anterior part of the tongue. The mouth was rinsed with tap water after each test. The taste panel sampled from lower concentration samples to higher. Each taster chose the first sample that could be sensed for sweetness. Sweetness potencies were reported relative to sucrose on a weight basis.

$\mathrm{N}$-Terminal amino acid sequencing and SDS-PAGE. For the recombinant wild-type brazzein and minor-form mutant, the 
$N$-terminal amino acid sequences were determined by automated Edman degradation using an Applied Biosystems 420A Protein sequencer. Denaturing SDS-PAGE was performed with $16.5 \%$ Tris-tricine gels to evaluate the purity of the purified recombinant wild-type brazzein and minor-form mutant. ${ }^{16}$ The molecular-mass markers were Polypeptide SDS-PAGE Molecular Weight Standards (Bio-Rad, Hercules, CA, USA) containing triosephosphate isomerase $(26.6 \mathrm{kDa})$, myoglobin $(17.0$ $\mathrm{kDa}), \alpha$-lactalbumin $(14.4 \mathrm{kDa})$, and aprotinin $(6.5 \mathrm{kDa})$. Coomassie Blue R-250 was used for protein staining.

Acknowledgments. This work was supported by the National Research Foundation of Korea Grant funded by the Korean Government (2010-0015724).

\section{References}

1. Nabors, L. O.; Gelardi, R. C. In Alternative Sweeteners; Marcel Dekker: New York, 1991; pp 151-171.

2. Ming, D.; Hellekant, G. FEBS Lett. 1994, 355, 106.
3. Assadi-Porter, F. M.; Aceti, D. J.; Cheng, H.; Markley, J. L. Arch. Biochem. Biophys. 2000, 376, 252.

4. Assadi-Porter, F. M.; Patry, S.; Markley, J. L. Protein Expr. Purif. 2008, $58,263$.

5. Lamphear, B. J.; Barker, D. K.; Brook, C. A.; Delaney, D. E.; Lane, J. R.; Beifuss, K.; Love, R.; Thompson, K.; Mayor, J.; Clough, R.; Harkey, R.; Poage, M.; Drees, C.; Horn, M. E.; Streatfield, S. J.; Nikolov, Z.; Woodard, S. L.; Hood, E. E.; Jilka, J. M.; Howard, J. A. J. Plant Biotechnol. 2005, 3, 103.

6. Berlec, A.; Jevnikar, Z.; Majhenic, A. C.; Rogelj, I.; Strukej, B. Appl. Microbiol. Biotechnol. 2006, 73, 158.

7. Malik, A.; Rudolph, R.; Söhling, B. Protein Expr. Purif. 2006, 47, 662.

8. Choi, J. H.; Lee, S. Y. Appl. Microbiol. Biotechnol. 2004, 64, 625.

9. Ying, H.; Liu, H. Immunol. Lett. 2007, 111, 66.

10. Assadi-Porter, F. M.; Maillet, E. L.; Radek, J. T.; Quijada, J.; Markley, J. L.; Max, M. J. Mol. Biol. 2010, 398, 584.

11. Guan, Z.; Hellekant, G.; Yan, W. Chem. Senses 1995, $20,701$.

12. Masuda, T.; Kitabatake, N. J. Biosci. Bioeng. 2006, 102, 375.

13. Koh, J. U.; Cho, H. Y.; Kong, K. H. Bull. Korean Chem. Soc. 2007, 28,772 .

14. Neu, H. C.; Heppel, L. A. J. Biol. Chem. 1965, 240, 3685.

15. Nossal, N. G.; Heppel, L. A. J. Biol. Chem. 1966, 241, 3055.

16. Schagger, H.; Von Jagoe, G. Anal. Biochem. 1987, 166, 368. 